\title{
Therapeutic utility of palmitoylethanolamide in the treatment of neuropathic pain associated with various pathological conditions: a case series
}

This article was published in the following Dove Press journal: Journal of Pain Research

25 October 2012

Number of times this article has been viewed

\section{Jan M Keppel Hesselink Thecla AM Hekker}

Institute for Neuropathic Pain, Bosch en Duin, Netherlands

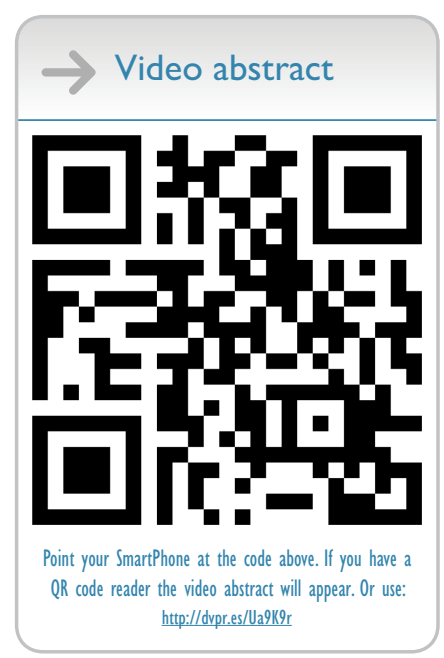

Correspondence: Jan M Keppel Hesselink Spoorlaan 2a, 3735 MV, Bosch en Duin, The Netherlands

Tel+3I 65I700527

Email jan@neuropathie.nu

\begin{abstract}
Palmitoylethanolamide (PEA), an endogenous fatty acid amide, has been demonstrated to bind to a receptor in the cell nucleus - the peroxisome proliferator-activated receptor - and performs a great variety of biological functions related to chronic and neuropathic pain and inflammation, as has been demonstrated in clinical trials. These include peripheral neuropathies such as diabetic neuropathy, chemotherapy-induced peripheral neuropathy, carpal tunnel syndrome, sciatic pain, osteoarthritis, low-back pain, failed back surgery syndrome, dental pains, neuropathic pain in stroke and multiple sclerosis, chronic pelvic pain, postherpetic neuralgia, and vaginal pains. Probably due to the fact that PEA is an endogenous modulator as well as a compound in food, such as eggs and milk, no serious side effects have been reported, nor have drug-drug interactions. This article presents a case series describing the application and potential efficacy and safety of PEA in the treatment of various syndromes associated with chronic pain that is poorly responsive to standard therapies.
\end{abstract}

Keywords: endocannabinoid, chronic, neuropathy, chemotherapy, itch, polyneuropathy

\section{Introduction}

Chronic pain and neuropathic pain are indications for which there is high need in the clinic, and as Loeser put it boldly recently, “... the lack of evidence for the outcomes of most of the things providers do for patients" is one of the preeminent crises in pain management today. ${ }^{1}$ Indeed, many patients suffering from neuropathic conditions have pain that is refractory to existing treatments. ${ }^{2}$ In this context, palmitoylethanolamide (PEA), an endogenous fatty acid amide, is emerging as a novel agent in the treatment of pain and inflammation. The compound was used many decades ago in some countries, but due to a lack of insight in its mechanism of action, interested weaned. Since the 1990s, interest has surged again due to the discovery of its effects in many different animal paradigms for pain and chronic inflammation. It is classified as a food for medical purposes or as a diet supplement in various countries of Europe. PEA has shown efficacy in many different preclinical animal models for chronic and neuropathic pain, and most importantly is effective in reducing pain in man in various clinical trials in a variety of pain states. ${ }^{3}$ The main target of PEA is thought to be the peroxisome proliferator-activated receptor alpha (PPAR- $\alpha$ ). ${ }^{4}$ This receptor is a regulator of gene networks which control pain and inflammation ${ }^{5}$, probably by switching off the nuclear factor-kappaB signaling cascade, ${ }^{4-7}$ a key element in the transcription of genes for proinflammatory mediators (cytokine, chemokines, nitric oxide). PEA also has affinity to cannabinoid-like $G$ protein-coupled receptors GPR55 and GPR119. ${ }^{8}$ PEA can influence ion channels (eg, potassium channels) that play a 
role in pain. ${ }^{9}$ Furthermore, PEA might desensitize transient receptor potential cation channel subfamily $\mathrm{V}$ member 1 channels on sensory neurons. ${ }^{10,11}$

PEA anti-inflammatory actions allowing for a reduction of peripheral and central sensitization are mediated via both neuronal and nonneuronal cells. The latter comprise glia (in particular microglia, which are the brain's macrophages) as well as peripheral and central mast cells. ${ }^{12-15}$ This profile of PEA may thus explain its broad potential in treating many different disorders related to pain and inflammation. ${ }^{16}$

Based on this knowledge, we selected a number of pain treatment-resistant patients and started adding PEA to the analgesic treatment regime. This paper presents seven different clinical cases, six of which showed a clear beneficial effect of PEA.

\section{Case presentations} Patient I

This was a 61-year-old Caucasian male suffering from metastatic prostate cancer. He has been receiving hormone therapy, estramustine, hyperthermia, and experimental dendritic cell therapy since 1996. In January 2009, his prostate-specific antigen count rose to $206 \mathrm{ng} / \mathrm{mL}$, and he suffered from bony metastases in the lumbar and pelvic area. He was subsequently treated with taxotere. Due to a relapse, in October 2009 an experimental protocol with five courses of the new chemotherapeutic compound sagopilone was started. Polyneuropathic side effects occurred from the second course of sagopilone; neuropathic pain in both feet and hands emerged and increased in intensity, characterized by allodynia and hyperalgesia, although the patient was treated with tramadol and pregabalin (up to $300 \mathrm{mg} /$ day). In December 2009, the patient was examined at our institution. His pain score was $7 / 10$ on the numeric rating scale (NRS), with periods of peak pain of 9 .

Treatment with PEA was initiated: PEA 600-mg tablets twice daily independent from meals. This treatment regime reduced pain quickly after 3 weeks to a mean NRS of 1-2. At this point, it was possible to continue chemotherapy with ongoing PEA administration. The patient's NRS remained low under methotrexate therapy for 3 months. In mid-2010, the analgesics tramadol and pregabalin were withheld and he was stable with virtually no pain, occasionally taking $500 \mathrm{mg}$ paracetamol.

\section{Patient 2}

This was a 62-year-old Caucasian male diagnosed with failed back surgery syndrome and a history of several surgical interventions for painful hernia (1980, 1991, 2004), including a neurolysis in 2006 after a skiing injury. Despite these surgical interventions, the patient suffered many years from severe chronic pain with dysesthesia, burning and hyperalgesia at the buttocks and right leg. Electromyography (2009) showed signs of denervation in L5 of the right leg. Clinically, there were no signs of motor dysfunction, but sensibility decreased and dysesthesia appeared in the innervation areas of L5 on the right leg. There were no reflex abnormalities. Treatment with gabapentin produced intolerable side effects before analgesia could happen. Diclofenac did not reduce pain. When the patient was examined at our institution, he complained about chronic pains and dysesthesia, scoring 7 on the NRS.

Treatment with PEA was initiated, $600 \mathrm{mg}$ twice daily. Within 3 weeks, pain as well as the burning sensations and dysesthesia decreased from NRS 7 to 4 and remained low until the last visit 1 month later.

\section{Patient 3}

This was a 54-year-old Caucasian man who had suffered for the past 7 years from type II diabetes, with a treatment regimen of metformin, glimepirid, and liraglutide. Because of hypertension, hypercholesterolemia, and stomach complaints, the patient also received hydrochlorthiazide, a statin, and losartan. The chronic neuropathic pain, especially prominent at both feet, scored 6 on the Douleur Neuropathique $4{ }^{17}$ scale and 7 on the NRS. His pain was characterized by burning and lancinating sensations and was largely unresponsive to gabapentin, duloxetine, capsaicine cream, pregabalin, and cannabis. He subsequently stopped all analgesic therapies. Treatment with PEA 600-mg tablets twice daily and R-alpha-lipoic acid (100 mg three times daily, a supplement explored extensively in diabetes mellitus type $\mathrm{II}^{18-20}$ ) was started, and pain dropped to NRS score 3 . When after 4 months the patient attempted to reduce the dose of PEA to $300 \mathrm{mg}$ twice daily, pain rose within a week to an NRS score of 8. Dosing was increased to PEA $600 \mathrm{mg}$ tablets twice daily, and again pain decreased to 3-4 within this period. Four months later, it was possible to decrease PEA dosing again to $300 \mathrm{mg}$ twice daily, without further increase of pain. In the following year, relapses of pain occurred, and treatment with PEA $600 \mathrm{mg}$ twice daily was able to reduce pain to a baseline NRS score of 3 again in about 10 days. Since then, the patient has been stable and continued taking PEA $300 \mathrm{mg}$ twice daily. It should be noted that PEA has no relevant affinity for either the $\mathrm{CB}_{1}$ or the $\mathrm{CB}_{2}$ receptor, explaining why this patient was a responder on PEA while a nonresponder on cannabis. 


\section{Patient 4}

This was a 64-year-old Caucasian male suffering from type II diabetes for 8 years, treated with metformin. Neuropathic pain in both feet started in 2011. Although the pain was treated with pregabalin ( $225 \mathrm{mg} /$ day) he still scored NRS 6 . We started prescribing according to our protocol for diabetic pains, consisting of $2000 \mathrm{IE}$ of vitamin $\mathrm{D}_{3}$, three times $100 \mathrm{mg}$ R-alpha-lipoic acid and twice daily PEA $600 \mathrm{mg}$, together with a topical cream twice daily consisting of adelmidrol and capsaicine (Algonerv). After 3 weeks, the patient felt much better, pain was reduced from 6 to 4 , and only in the morning did some burning pain remain. After 3 months of treatment, pain was reduced to 1.5 , although the patient forgot to use the alpha-lipoic acid. He was able to resume full activities as an industrial designer and was working with much pleasure on a new machine.

\section{Patient 5}

This was a 66-year-old Caucasian male suffering from chronic idiopathic axonal polyneuropathy, showing a mean pain score of 8 on the NRS. The diagnosis was supported by electromyographic analysis in 2011, showing signs of a severe axonal neuropathy, without any sign of demyelinization. The complaints started in 2000 with burning and tingling in both feet. Neuropathic pain started after walking 10 miles and more. Being an enthusiastic walker, he started to feel very annoyed by the symptoms. He refused to take analgesics such as amitriptyline and pregabalin because of much-feared side effects. We started treatment with PEA according to our protocol, and month by month his pain decreased. After 2-3 months, pain was reduced to NRS $1-2$ and the patient was able to start walking long distances again.

\section{Patient 6}

This was a still-very-active 81-year-old Caucasian female with 25 years' vaginal complaints from lichen sclerosis. She underwent a partial vulvectomy in 2008 because of a lesion that showed vulvar intraepithelial neoplasia II. Her pain fluctuated from no pain at all to a 7 on the NRS. She also had one or more lesions that came and went and a very fierce itch. Her family doctor and several gynecologists and dermatologists advised and prescribed petroleum jelly, vitamin $\mathrm{E}$ and zinc ointments, triamcinolone acetenoide, several other local corticosteroid ointments, and several local and systemic yeast treatments. By the end of 2010, passing urine had become very painful and she could not ride her bike anymore. Her gynecologist prescribed a very strong local corticosteroid, clobetasol, after which the pain worsened to $9-10$ on the
NRS. The patient became desperate and asked for advice in our clinic. She was unable to ride her bicycle and her quality of life was severely compromised. She started with PEA according to our protocol, and in addition to the oral PEA, we prescribed a twice-daily local vaginal gel containing a precursor of palmitoylethanolamide, adelmidrol (Saginil gel). The next day, the pain and itching almost disappeared. She started cycling again. She used the gel intermittently during periods of several weeks. The improvements in pain and itching lasted around 6 months. Unfortunately, in the second half of 2011, lesions reappeared. Another biopsy was performed, and her lichen sclerosis had progressed to vulva carcinoma. A radical vulvectomy and selective inguinal lymphadenectomy were performed. Histopathologic examination showed no metastasis. All complaints were gone.

\section{Patient 7}

This was an 80-year-old Caucasian female with complaints of pain in feet and legs, low-back pain and paresthesia, as well as numbness. The complaints started 3 years earlier, when electromyography showed signs of a slight axonal neuropathy. No sign of a stenosis of the lumbar region was evident by computed tomography scan, and chronic idiopathic axonal polyneuropathy was diagnosed. Because of her advanced age, the patient did not wish to take amitriptyline and related compounds. Her pain score was 6.5 on the NRS; treatment with topical amitriptyline cream was ineffective. PEA $600 \mathrm{mg}$ twice daily was added; however, no decrease of pain was observed. The addition of other prescribed supplements, such as acetyl-L-carnitine, did not decrease pain either. She is presented as a nonresponder. One of the reasons for this might have been advanced age combined with a nonspecific chronic pain.

\section{Discussion}

Neuropathic pain is challenging to manage, and many patients experience pain that is refractory to existing treatments. Physicians usually start treatment for chronic neuropathic pain with monotherapy. In this protocol, starting dosage is low with most of the available drugs until titration, then, according to the subjective response, dosage is increased to the maximum tolerated. When monotherapy proves insufficient, combinations of various pharmacologic compounds follow, combining serotonin-noradrenaline reuptake inhibitors, tricyclic antidepressants, anticonvulsants, opioids, natural and synthetic derivatives from cannabis, and topical analgesics. ${ }^{21-24}$ Until now, however, no consistent improvement in the treatment of neuropathic pain has been 
achieved, and recent clinical trials of older analgesics seem even to show decreased efficacy. ${ }^{25}$ There is clearly an urgent need for new, innovative compounds that are not only effective but have a favorable profile in terms of side effects and drug-drug interactions. Not only does PEA display these characteristics, but the case series reported here suggest PEA's therapeutic potential for different chronic and neuropathic pain states. Indeed, in these cases, PEA - alone or added to standard analgesics - was able to reduce pain by $40 \%-80 \%$ compared to baseline scores on the NRS. The initial PEA-induced clinical relevant pain reduction was often seen between week 1 and week 3 . However, in some cases the response only emerged after 5 weeks of treatment. Occasionally, as in the last case described, nonresponse to PEA occurs.

\section{PEA to protect against chemotherapy- induced painful polyneuropathy}

Chemotherapy-induced neuropathic pain (patient 1) must be taken into account for both the associated disability and distress, but also to optimize the chemotherapy itself. Recently, the effect of PEA on pain and nerve functions in patients with chemotherapy-induced painful neuropathy was assessed. ${ }^{26}$ In brief, 20 patients undergoing thalidomide plus bortezomib treatment for multiple myeloma received $300 \mathrm{mg}$ PEA twice daily for 2 months, starting after chemotherapy-induced neurotoxicity was established. Although no variable returned to normal, pain and all neurophysiological measures - assessing $\mathrm{A} \alpha, \mathrm{A} \beta$, and $\mathrm{A} \delta$ fibers - significantly improved. These results suggest a possible neuroprotective effect of PEA on myelinated nerve fibers. Importantly, concomitant PEA treatment allowed the maintenance of chemotherapeutic dose without further deterioration of nerve functions. The ability to continue chemotherapy in patients suffering from neuropathic pain, instead of stopping or reducing therapy, would clearly impact positively on survival rates also. A multicenter double-blind study evaluating the efficacy and safety of PEA in chemotherapy-induced neuropathic pain is currently in progress.

\section{PEA treatment schedule}

We adhered to a standardized dosing regimen of PEA in cases of neuropathic pain, independent of its etiology or pathogenesis, starting with twice-daily sublingual PEA $600 \mathrm{mg}$, for quick absorption and to avoid first-pass effects. After 10 days, we switched to tablets of $600 \mathrm{mg}$ PEA twice daily, and if pain was sufficiently reduced, the dose was reduced to $300 \mathrm{mg}$ twice daily. We developed this treatment schedule after treating hundreds of neuropathic pain patients. Based on our clinical experience, we hypothesize that starting with the sublingual form of PEA for at least 10 days might help to quickly reach sufficient therapeutic levels of PEA. This of course has never been investigated and is only a clinical impression. After the initial phase of 10 days, treatment with normal tablets can start. Due to the first-pass effects, the bioavailability of the tablets might be somewhat less compared to the sublingual form. However, formal pharmacokinetic and bioavailability studies are lacking.

\section{PEA as part of multimodal pharmacotherapy}

In our case series, combinations with regular analgesics did not lead to drug-drug interactions, and in all seven patients PEA tolerability was excellent. As all patients were treatmentresistant to previous analgesics, we suppose the good clinical response is more than placebo alone. To date, we have treated in our clinic around 1000 patients suffering from neuropathic pain with PEA, sometimes as monotherapy, but mostly combined with topical analgesic creams consisting of amitriptyline (5\% and $10 \%)$, racemic ketamine $(10 \%)$, baclofen $(5 \%)$, or gabapentin $(10 \%) .{ }^{27-29}$ Treatment duration varied from several weeks up to 2 years. Combination of PEA with analgesics such as tramadol, pregabalin, gabapentin, and duloxetine in our hands has never led to any discomfort or adverse interactions. The combination of PEA, sometimes with other supplements such as alpha-lipoic acid and topical analgesic creams, results in sufficient to good pain reduction in neuropathic pain patients, without serious side effects and with good tolerability. The only side effects of PEA we have seen were related to a feeling of heaviness in the stomach after the tablets were taken and rarely gastrointestinal discomfort and diarrhea after the sublingual PEA formulation, most probably due to the presence of sorbitol as a sweetener. These side effects were rarely a reason for stopping treatment. The fact that PEA is an endogenous modulator and not a synthetic molecule, together with the fact that PEA mainly works via the PPAR- $\alpha$ receptor and not, as far as we know, via the PPAR- $\gamma$ receptor (also known as the glitazone receptor), explains the absence of side effects known for compounds such as glitazone and ploglitazone.

Promising results for PEA have been reported in various clinical trials in which the compound was evaluated for efficacy and safety. In a pivotal randomized, double-blind, placebo-controlled clinical trial of 636 patients with sciatic pain, a significant and clinically relevant analgesic effect was documented for $300 \mathrm{mg}$ and $600 \mathrm{mg}$ PEA. ${ }^{30}$ Similar results 
were obtained in a randomized clinical trial with 85 chronic sciatic patients. ${ }^{31}$ Quality of life and functionality related to the back improved significantly in favor of the PEA groups in both studies. Various smaller studies evaluating the effect of PEA in other pain states also demonstrated safety and efficacy. ${ }^{32-36}$ To date, more than 20 positive clinical trials with PEA have been presented and reviewed in detail, with a total of nearly 2000 patients treated. ${ }^{3}$ No side effects of note have been reported, nor have there been any deleterious drug-drug interactions. ${ }^{3}$ In a recent case series of severe central neuropathic pain in King Charles spaniels, PEA also reduced pain as well as inflammation of the eyes. This clearly supports the analgesic as well as the anti-inflammatory properties of PEA. PEA may thus represent a promising addition to our analgesic regimen for the treatment of man as well as for companion animals, with potential for good tolerability. Worldwide, more than 800,000 patients have been treated with PEA, most of them in Italy and Spain, with some in The Netherlands, Germany, UK, Canada and the US, and no serious side effects have been reported (Epitech, data on file).

\section{Conclusion}

PEA represents a promising addition to our therapeutic armamentarium for neuropathic pain, with potential for good tolerability and a low propensity for side effects.

\section{Disclosure}

The authors report no conflicts of interest in this work.

\section{References}

1. Loeser JD. Five crises in pain management. Pain Clin Updates. 2012;20: $1-4$.

2. Dworkin RH, O'Connor AB, Audette J, et al. Recommendations for the pharmacological management of neuropathic pain: an overview and literature update. Mayo Clin Proc. 2010;85:S3-S14.

3. Keppel Hesselink JM. New targets in pain, non-neuronal cells, and the role of palmitoylethanolamide. Open Pain J. 2012;5:12-23.

4. LoVerme J, Russo R, La Rana G, et al. Rapid broad-spectrum analgesia through activation of peroxisome proliferator-activated receptor-alpha. J Pharmacol Exp Ther. 2006;3:1051-1061.

5. Lo Verme J, Fu J, Astarita G, et al. The nuclear receptor peroxisome proliferator-activated receptor-alpha mediates the anti-inflammatory actions of palmitoylethanolamide. Mol Pharmacol. 2005;67:15-19.

6. Darmani NA, Izzo AA, Degenhardt B, et al. Involvement of the cannabimimetic compound, $\mathrm{N}$-palmitoylethanolamine, in inflammatory and neuropathic conditions. A review of the available preclinical data and first human studies. Neuropharmacology. 2005;48:1154-1163.

7. Genovese T, Esposito E, Mazzon E, et al. Effects of palmitoylethanolamide on signaling pathways implicated in the development of spinal cord injury. J Pharmacol Exp Ther. 2008;326:12-23.

8. Naderi N, Majidi M, Mousavi Z, Khoramian Tusi S, Mansouri Z. The interaction between intrathecal administration of low doses of palmitoylethanolamide and AM251 in formalin-induced pain related behavior and spinal cord IL1- $\beta$ expression in rats. Neurochem Res. 2012;37: $778-785$.
9. Yoshihara S, Morimoto H, Ohori M, Yamada Y, Abe T, Arisaka O. Cannabinoid receptor agonists inhibit $\mathrm{Ca}(2+)$ influx to synaptosomes from rat brain. Pharmacology. 2006;76:157-162.

10. Ho WS, Barrett DA, Randall MD. 'Entourage' effects of $\mathrm{N}$-palmitoylethanolamide and $\mathrm{N}$-oleoylethanolamide on vasorelaxation to anandamide occur through TRPV1 receptors. Br J Pharmacol. 2008;6: $837-846$.

11. Appendino G, Ligresti A, Minassi A, et al. Conformationally constrained fatty acid ethanolamides as cannabinoid and vanilloid receptor probes. J Med Chem. 2009;52:3001-3009.

12. Loría F, Petrosino S, Mestre L, et al. Study of the regulation of the endocannabinoid system in a virus model of multiple sclerosis reveals a therapeutic effect of palmitoylethanolamide. Eur J Neurosci. 2008;28: 633-641.

13. Esposito E, Paterniti I, Mazzon E, et al. Effects of palmitoylethanolamide on release of mast cell peptidases and neurotrophic factors after spinal cord injury. Brain Behav Immun. 2011;25:1099-1112.

14. Cerrato S, Brazis P, della Valle MF, Miolo A, Puigdemont A. Effects of palmitoylethanolamide on immunologically induced histamine, PGD2 and TNF-alpha release from canine skin mast cells. Vet Immunol Immunopathol. 2010;133:9-15.

15. De Filippis D, Luongo L, Cipriano M, et al. Palmitoylethanolamide reduces granuloma-induced hyperalgesia by modulation of mast cell activation in rats. Mol Pain. 2010;10:7:3

16. Keppel Hesselink JM. Glia as a new target for neuropathic pain, clinical proof of concept for palmitoylethanolamide, a glia modulator. Anesth Pain Intensive Care. 2011;15:143-145.

17. Bouhassira D, Attal N, Alchaar H, et al. Comparison of pain syndromes associated with nervous or somatic lesions and development of a new neuropathic pain diagnostic questionnaire (DN4). Pain. 2005;114: 29-36.

18. Ziegler D, Nowak H, Kempler P, Vargha P, Low PA. Treatment of symptomatic diabetic polyneuropathy with the antioxidant alpha-lipoic acid: a meta-analysis. Diabet Med. 2004;21:114-121.

19. Ziegler D, Ametov A, Barinov A, et al. Oral treatment with alpha-lipoic acid improves symptomatic diabetic polyneuropathy: the SYDNEY 2 trial. Diabetes Care. 2006;29:2365-2370.

20. Ruessmann HJ. Switching from pathogenetic treatment with alpha-lipoic acid to gabapentin and other analgesics in painful diabetic neuropathy: a real-world study in outpatients. J Diabetes Complications. 2009;23: $174-177$.

21. Vorobeychik Y, Gordin V, Mao J, et al. Combination therapy for neuropathic pain: a review of current evidence. CNS Drugs. 2011;25: 1023-1034.

22. Thaler A, Gupta A, Cohen SP. Cannabinoids for pain management. Adv Psychosom Med. 2011;30:125-138.

23. Jaggi AS, Singh N. Therapeutic targets for the management of peripheral nerve injury-induced neuropathic pain. CNS Neurol Disord Drug Targets. 2011;10:589-609.

24. Kopsky DJ, Keppel Hesselink JM. High doses of topical amitriptyline in neuropathic pain: two cases and literature review. Pain Pract. 2011;12: $148-153$.

25. Finnerup NB, Sindrup SH, Jensen TS. The evidence for pharmacological treatment of neuropathic pain. Pain. 2010;150:573-581.

26. Truini A, Biasiotta A, Di Stefano G, et al. Palmitoylethanolamide restores myelinated-fibre function in patients with chemotherapyinduced painful neuropathy. CNS Neurol Disord Drug Targets. 2011;10: 916-920.

27. Kopsky DJ, Keppel Hesselink JM. Multimodal stepped care approach involving topical analgesics for severe intractable neuropathic pain in CRPS type 1: a case report. Case Report Med. 2011;2011:319750.

28. Liebregts R, Kopsky DJ, Keppel Hesselink JM. Topical amitriptyline in post-traumatic neuropathic pain. J Pain Symptom Manage. 2011;41: e6-e8.

29. Keppel Hesselink JM, Kopsky DJ. Intractable neuropathic pain due to ulnar nerve entrapment treated with cannabis and ketamine $10 \%$. J Clin Anesth. 2012;24:78-79. 
30. Guida G, de Martino M, de Fabiani A, et al. La palmitoiletanolamida (Normast) en el dolor neuropatico cronico por lumbociatalgia de tipo compresivo: estudio clinico multicentrico. [Palmitoylethanolamide treatment of sciatic pain: results form a multicenter study]. Dolor. 2010;25:35-42.

31. Canteri L. Reduction of analgesics in patients suffering from lumbosciatic pain, treated with palmitoylethanolamide. Dolor. 2010;25: 227-234.

32. Assini A, Laricchia D, Pizzo R, et al. The carpal tunnel syndrome in diabetes: clinical and electrophysiological improvement after treatment with palmitoylethanolamide. Eur J Neurol. 2010;17:295.

33. Biasiotta A, La Cesa S, Leone C, Di Stefano G, Truini A, Cruccu G. Efficacy of palmitoylethanolamide in patients with painful neuropathy. A clinical and neurophysiological open study. Preliminary results. Eur J Pain Suppl. 2010;4:77.
34. Bortolotti F, Russo M, Bartolucci ML, Alessandri Bonetti G, Gatto MR, Marini I. Palmitoylethanolamide vs NSAID in the treatment of TMJD pain. J Orofac Pain. In press 2012.

35. Phan NQ, Siepmann D, Gralow I, Ständer S. Adjuvant topical therapy with a cannabinoid receptor agonist in facial postherpetic neuralgia. J Dtsch Dermatol Ges. 2010;8:88-91.

36. Desio P. Combination of pregabalin and palmitoylethanolamide (PEA) for neuropathic pain treatment. Pathos. 2010;17:9-14.

Journal of Pain Research

\section{Publish your work in this journal}

The Journal of Pain Research is an international, peer-reviewed, open access, online journal that welcomes laboratory and clinical findings in the fields of pain research and the prevention and management of pain. Original research, reviews, symposium reports, hypothesis formation and commentaries are all considered for publication.

The manuscript management system is completely online and includes a very quick and fair peer-review system, which is all easy to use. Visit http://www.dovepress.com/testimonials.php to read real quotes from published authors. 